\title{
Mental health needs of deaf and hearing impaired children and adolescents
}

\author{
Andrew Clark
}

Diagnosis and treatment of mental health problems in individuals suffering from deafness or severe hearing impairment poses particular challenges. This is recognised for adults by the provision of specialised services based in London, Birmingham and Manchester but for children and adolescents there is only a specialised out-patient service based at Springfield Hospital in London which has itself only been in existence two years. This day conference of invited hearing and nonhearing professionals held in Birmingham in October 1994 considered how appropriate services might further develop.

Dr Peter Hindley outlined the work of the Springfield Unit which comprises a consultant child psychiatrist ( $\mathrm{PH}$ ), a community psychiatric nurse, a social worker, a counsellor and an administrator. It provides out-patient and consultation services primarily to southern England. He presented data relating to their first 130 referrals. Of these, $90 \%$ were suffering from profound or severe deafness and $60 \%$ reliant solely upon signing for communication. Conduct and emotional disorders were common but a number suffered obsessive-compulsive disorder (2.3\%), schizophrenia (1.6\%), depressive disorders (3.9\%), bipolar disorders $(2.3 \%)$, or eating disorders $(2.3 \%)$. Treatments as appropriate (including consultation) were offered within a systemic framework and around $66 \%$ of children showed significant improvement. Very few young people required pharmacotherapy $(5 \%)$ or in-patient care $(2 \%)$ but when this latter was necessary it was in a hearing child and adolescent psychiatric unit. Although not ideal, much of the feared isolation was overcome by support from signing staff from the out-patient service and by provision of an interpreter when necessary.

Dr Tlehjo van Gent from Leiden, Holland, then described the development of a ten- bedded in-patient unit specifically for deaf children and adolescents. Planning began in 1985 but the service was only now becoming fully functional, having commenced in 1992. Consequently it was still awaiting full evaluation but he considered they had already learnt some important lessons. These included the need to recruit both deaf staff and skilled signing hearing staff, to include qualified interpreters within the establishment, and to allow much greater time for both assessments and therapeutic change to take place. Working with deaf children and their families requires extra time and staff which doubles the costs when compared with services for hearing children. Recruiting deaf staff is essential but this may mean high training costs due to a lack of initially suitably qualified candidates. The physical environment is also important needing minimal distractions to signing, good lighting and round tables to aid visual contact.

Participants spent the afternoon in workshops considering these issues and possible future developments, before reuniting for a plenary session. The major theme was for a 'bicultural service' with hearing and non-hearing professionals working in partnership. There was concern at the fragmentary nature of current provision and an emphasis on developing geographically accessible and coordinated services, initially increasing out-patient availability but moving towards development of in-patient facilities. Fuller details of the conference proceedings are available from Dr Margaret de Feu of the South Birmingham Mental Health (NHS) Trust.

Andrew Clark, Consultant Adolescent Psychiatrist. The Irwin Unit for Young People, Joseph Sheldon Hospital, Rubery. Birmingham B45 9BA 